\title{
Effects of belimumab, a B lymphocyte stimulator-specific inhibitor, on disease activity across multiple organ domains in patients with systemic lupus erythematosus: combined results from two phase III trials
}

\author{
Susan Manzi, ${ }^{1}$ Jorge Sánchez-Guerrero, ${ }^{2}$ Joan T Merrill, ${ }^{3}$ Richard Furie, ${ }^{4}$ Dafna Gladman, ${ }^{5}$ \\ Sandra V Navarra, ${ }^{6}$ Ellen M Ginzler, ${ }^{7}$ David P D'Cruz, ${ }^{8}$ Andrea Doria, ${ }^{9}$ Simon Cooper, ${ }^{10}$ \\ Z John Zhong, ${ }^{10}$ Douglas Hough, ${ }^{10}$ William Freimuth, ${ }^{10}$ Michelle A Petri ${ }^{11}$ \\ on behalf of the BLISS-52 and BLISS-76 Study Groups
}

${ }^{1}$ Allegheny Singer Research Institute, West Penn Allegheny Health System, Temple

University School of Medicine, Pittsburgh, Pennsylvania, USA ${ }^{2}$ Instituto Nacional de Ciencias Medicas y Nutricion "Salvador Zubiran," Delegacion Tlalpan, Mexico City, Mexico ${ }^{3}$ Oklahoma Medical Research Foundation, Oklahoma City, Oklahoma, USA

${ }^{4}$ North Shore-LIJ Health System, Lake Success, New York, USA

${ }^{5}$ Toronto Western Hospital, Toronto, Canada

${ }^{6}$ University of Santo Tomas Hospital, Manila, Philippines ${ }^{7}$ SUNY Downstate Medical Center, Brooklyn, New York USA

${ }^{8}$ St Thomas' Hospital, London, UK

9University of Padova, Padova, Italy

${ }^{10}$ Human Genome Sciences, Rockville, Maryland, USA

11Johns Hopkins University, School of Medicine, Baltimore, Maryland, USA

\section{Correspondence to} Susan Manzi, Allegheny Singer Research Institute, West Penn Allegheny Health System, Temple University School of Medicine, Pittsburgh, Pennsylvania 15224, USA; smanzi@wpahs.org

Accepted 25 February 2012 Published Online First 11 May 2012

\begin{abstract}
Objective To evaluate the effects of belimumab versus placebo, plus standard systemic lupus erythematosus (SLE) therapy, on organ domain-specific SLE disease activity.

Methods Data obtained after 52 weeks of treatment from two phase III trials (BLISS-52 and BLISS-76) comparing belimumab 1 and $10 \mathrm{mg} / \mathrm{kg}$ versus placebo, plus standard therapy, in 1684 autoantibody-positive patients were analysed post hoc for changes in British Isles Lupus Assessment Group (BILAG) and Safety of Estrogens in Lupus National Assessment-Systemic Lupus Erythematosus Disease Activity Index (SELENA-SLEDAI) organ domain scores.
\end{abstract}

Results At baseline, the domains involved in the majority of patients were musculoskeletal and mucocutaneous by both BILAG and SELENA-SLEDAI, and immunological by SELENA-SLEDAI. At 52 weeks, significantly more patients treated with belimumab versus placebo had improvement in BILAG musculoskeletal and mucocutaneous domains (1 and 10 $\mathrm{mg} / \mathrm{kg}$ ), and in SELENA-SLEDAI mucocutaneous (10 mg/ $\mathrm{kg}$ ), musculoskeletal (1 mg/kg) and immunological (1 and $10 \mathrm{mg} / \mathrm{kg}$ ) domains. Improvement was also observed in other organ systems with a low prevalence $(\leq 16 \%)$ at baseline, including the SELENA-SLEDAI vasculitis and central nervous system domains. Significantly fewer patients treated with belimumab versus placebo had worsening in the BILAG haematological domain $(1 \mathrm{mg} / \mathrm{kg})$ and in the SELENA-SLEDAl immunological $(10 \mathrm{mg} / \mathrm{kg})$, haematological $(10 \mathrm{mg} / \mathrm{kg})$ and renal $(1 \mathrm{mg} / \mathrm{kg})$ domains.

Conclusions Belimumab treatment improved overall SLE disease activity in the most common musculoskeletal and mucocutaneous organ domains. Less worsening occurred in the haematological, immunological and renal domains.

Systemic lupus erythematosus (SLE) is a heterogeneous autoimmune disease associated with considerable morbidity, increased mortality and poor health-related quality of life. ${ }^{12}$ The assessment of the degree of organ system involvement is fundamental for determining the burden of disease, as well as response to treatment.
Belimumab is a human immunoglobulin G1 $\lambda$ monoclonal antibody that inhibits B-cell survival and differentiation by neutralising soluble B lymphocyte stimulator; ${ }^{3}$ B lymphocyte stimulator is overexpressed in patients with SLE and correlates with changes in disease activity. ${ }^{4}$ Two international phase III trials, BLISS-52 and BLISS-76, have evaluated the safety and efficacy of belimumab in patients with seropositive SLE (defined as positive antinuclear antibody or anti-ds DNA). ${ }^{5,}{ }^{\circ}$ In both trials, belimumab $10 \mathrm{mg} / \mathrm{kg}$ plus standard SLE therapy met the primary endpoint of significantly higher SLE Responder Index (SRI) response rates at week 52 than with placebo plus standard SLE therapy. In addition, a multivariate analysis of the combined clinical data from both BLISS trials indicated that certain baseline characteristics of patients with SLE (eg, high disease activity [Safety of Estrogens in Lupus National Assessment-Systemic Lupus Erythematosus Disease Activity Index (SELENASLEDAI) score $\geq 10]$, anti-dsDNA positivity, low complement levels and prednisone use) were associated with a greater response to belimumab compared with standard therapy alone as measured by the SRI response at week 52 . $^{\text {? }}$

The SRI is a composite responder index that includes one measure of disease activity improvement (ie, $\geq 4$-point decrease in SELENA-SLEDAI). ${ }^{8}$ Also included in the SRI are two measures to ensure that improvement in disease activity is not offset by worsening of the patient's overall health status (ie, <0.3-point increase from baseline in the Physician's Global Assessment score) or by worsening of disease in organ systems (ie, no new British Isles Lupus Assessment Group (BILAG) A domain score or no more than one new B score). In the present exploratory analysis, the effects of 1 year of belimumab treatment on organ domains, as assessed by the SELENA-SLEDAI ${ }^{9}$ and BILAG ${ }^{10}$ scoring systems, were analysed in patients participating in the two phase III trials.

\section{METHODS}

BLISS-52 $(n=865)$ and BLISS-76 $(n=819)$ were randomised, double-blind, placebo controlled, 
international, multicentre trials that compared belimumab 1 and $10 \mathrm{mg} / \mathrm{kg}$ plus standard SLE therapy with placebo plus standard therapy in patients with seropositive SLE. The trials had similar designs, which have been described previously. ${ }^{6}$ In brief, at screening all patients had a SELENA-SLEDAI score of 6 or greater, were seropositive for antinuclear antibody $(\geq 1: 80)$ and/ or anti-dsDNA antibody ( $\geq 30 \mathrm{IU} / \mathrm{ml})$, and had received a stable regimen of standard therapy for 30 days or more before the study. Standard therapy was not protocol defined; rather, it consisted of background medications (including corticosteroids, and immunosuppressive and antimalarial agents) previously chosen by the physician for individual patients that did not violate protocol restrictions.

Belimumab or placebo was infused intravenously on days 0 , 14 and 28, and then every 28 days to week 48 (BLISS-52) or week 72 (BLISS-76). ${ }^{5}$ Patients with severe active lupus nephritis or severe active central nervous system (CNS) manifestations were excluded from the studies. Changes in concomitant corticosteroid, immunosuppressive and antimalarial medications were progressively restricted over the course of both trials. The primary endpoint in both trials, SRI response rate at week 52 , was an evaluation of treatment effect. The trial was not designed or powered to determine efficacy in any specific organ system. No organ system manifestations, other than serological activity (immunological), were enriched a priori in the studies. Efficacy assessments included changes in SELENA-SLEDAI and BILAG scores every 4 weeks. As the two phase III trials were similar in design and the primary endpoint was met in both, data across the trials were pooled in this post-hoc analysis to increase precision.

SELENA-SLEDAI domains were determined based on the SLEDAI analysis of Bombardier et a ${ }^{11}$ in which the 24 individual items are grouped into nine organ systems including dermal, which is henceforth described as mucocutaneous for comparison with the similar BILAG domain. Improvement in SELENA-SLEDAI organ domains was defined as a reduction in total domain scores in patients with organ domain involvement at baseline, whereas worsening was defined as any increase in scores in patients with or without organ domain involvement at baseline. The proportions of patients who improved or worsened in each of the organ domains from baseline to week 52 were summarised by treatment group. In addition, the improvements in SELENA-SLEDAI organ domains were evaluated by an adjusted mean SELENA-SLEDAI analysis over 52 weeks. ${ }^{12}$ The adjusted mean SELENA-SLEDAI is equivalent to the area under the curve of SELENA-SLEDAI over time divided by the time interval.

The BILAG organ systems were determined by the classic BILAG index. ${ }^{10}$ Organ domain involvement by BILAG was defined as an A or B score. Improvement in BILAG organ domains during the study was defined as a step down to a B, C or $\mathrm{D}$ score in patients with an $\mathrm{A}$ or $\mathrm{B}$ score at baseline; worsening was defined as a step up in score from B, C, D or E at baseline to an $\mathrm{A}$ or $\mathrm{B}$ score. In addition to the summaries of the proportions with improvement and worsening, the proportion of patients who improved one step (eg, improvement from A to $B$ or $B$ to $C$ ) or three steps (eg, improvement from $A$ to $D$ ) in a given organ domain from $\mathrm{A}$ or $\mathrm{B}$ at baseline to week 52 was evaluated.

For both BILAG and SELENA-SLEDAI, patients who withdrew from the study or received protocol-prohibited medications were considered to have no improvement in the improvement analyses, and missing data were analysed using the last observation carried forward method in the worsening analyses. As the studies were not designed to examine the treatment effect of belimumab on individual organ domains, all reported $p$ values are nominal. Improvement and worsening rates were compared between each of the belimumab groups and the placebo group using logistic regression adjusted by study effect. For organ domains with low event counts $(n \leq 5)$, the analysis was performed using Fisher's exact test.

\section{RESULTS}

\section{Baseline characteristics}

Baseline demographics and disease characteristics were similar across treatment groups and between BLISS-52 and BLISS-76 (table 1). ${ }^{5}$ Among all 1684 patients, the mean age was 37.8 years, $94.1 \%$ were women and $47.4 \%$ were Caucasian. The mean SELENA-SLEDAI score was 9.71 , and $60.8 \%$ of patients had one or more BILAG A or two or more BILAG B organ domain scores. Baseline organ domain involvement based on BILAG and SELENA-SLEDAI scoring was also similar across the three treatment groups and between the two trials (figure 1). In all, $92 \%$ of patients had a BILAG A or B domain score at baseline; $94 \%$ had involvement of two or more SELENA-SLEDAI organ domains and $57 \%$ had three or more organ systems involved. The most frequently affected organ domains were musculoskeletal (BILAG 59.9\%; SELENA-SLEDAI 65.4\%), mucocutaneous (BILAG 58.8\%; SELENA-SLEDAI $81.9 \%$ ) and immunological (SELENA-SLEDAI 79.5\%). CNS and renal involvement were relatively uncommon at baseline because of the exclusion of patients with severe active lupus nephritis or severe active CNS manifestations. In the SELENA-SLEDAI analyses, all patients with vascular domain involvement had vasculitis.

\section{Efficacy}

The primary endpoint was achieved in both BLISS-52 and BLISS-76, as shown by a significantly higher SRI response rate at 52 weeks with belimumab plus standard therapy than with placebo plus standard therapy. ${ }^{5}$ The SRI response rates in BLISS-52 were $43.6 \%$ with placebo versus $51.4 \%(\mathrm{p}=0.01)$ and $57.6 \%(\mathrm{p}<0.001)$ with belimumab 1 and $10 \mathrm{mg} / \mathrm{kg}$, respectively; the corresponding rates in BLISS-76 were $33.5 \%$ versus $40.6 \%(p=0.09)$ and $43.2 \%(p=0.02)$. The SRI response rates for both studies combined were $38.6 \%$ with placebo versus $46.2 \%$ $(\mathrm{p}=0.005)$ and $50.6 \%(\mathrm{p}<0.001)$ with belimumab 1 and $10 \mathrm{mg} /$ $\mathrm{kg}$, respectively. By pooled analysis, patients treated with belimumab also had significant improvements in the components of the SRI endpoint: the proportions with a 4-point or greater reduction in the SELENA-SLEDAI score at week 52 were $40.7 \%$ with placebo versus $48.1 \%(p=0.006)$ and $52.6 \% \quad(p<0.001)$ with belimumab 1 and $10 \mathrm{mg} / \mathrm{kg}$, respectively; the corresponding proportions with no new BILAG A or no more than one new B score at week 52 were $69.4 \%$ versus $76.7 \%(p=0.005)$ and $75.5 \%(p=0.02)$; and the corresponding proportions with no worsening in the physician's global assessment score at week 52 were $66.2 \%$ versus $75.8 \%(p=0.003)$ and $75.0 \%(p=0.001)$.

\section{Organ domain improvement and worsening}

As assessed by the BILAG index, the differences observed between belimumab and placebo regarding improvement or worsening in organ domains occurred primarily in the domains most commonly affected at baseline. At 52 weeks, significantly more patients in both belimumab groups had improvement in the musculoskeletal and mucocutaneous BILAG domains (figure $2 \mathrm{~A}$ ). Figure $2 \mathrm{~B}$ shows the proportions of patients improving from BILAG A or B scores at baseline to B, C or D scores at week 
Table 1 Demographics, disease characteristics and concomitant SLE medications at baseline

\begin{tabular}{|c|c|c|c|}
\hline & $\begin{array}{l}\text { BLISS-52 } \\
(\mathrm{n}=865)\end{array}$ & $\begin{array}{l}\text { BLISS-76 } \\
(\mathrm{n}=819)\end{array}$ & $\begin{array}{l}\text { Pooled } \\
\text { data } \\
(n=1684)\end{array}$ \\
\hline \multicolumn{4}{|l|}{ Demographics } \\
\hline Sex, female, $\%$ & 94.9 & 93.3 & 94.1 \\
\hline Age, years, mean $\pm S D$ & $35.5 \pm 11.1$ & $40.2 \pm 11.5$ & $37.8 \pm 11.5$ \\
\hline \multicolumn{4}{|l|}{ Ethnicity, \% } \\
\hline White & 26.5 & 69.5 & 47.4 \\
\hline Asian & 37.8 & 3.4 & 21.1 \\
\hline Black & 3.5 & 14.4 & 8.8 \\
\hline \multicolumn{4}{|l|}{ Disease characteristics } \\
\hline SLE disease duration, years, mean $\pm S D$ & $5.31 \pm 5.32$ & $7.52 \pm 7.10$ & $6.38 \pm 6.35$ \\
\hline SELENA-SLEDAI score, mean \pm SD & $9.75 \pm 3.76$ & $9.67 \pm 3.75$ & $9.71 \pm 3.76$ \\
\hline \multicolumn{4}{|l|}{ BILAG organ domain involvement } \\
\hline$\geq 1 \mathrm{~A}$ or $\geq 2 \mathrm{~B}$ scores, $\%$ & 58.3 & 63.5 & 60.8 \\
\hline$\geq 1$ A score, $\%$ & 19.0 & 12.1 & 15.6 \\
\hline \multicolumn{4}{|l|}{ Medication, \% } \\
\hline \multicolumn{4}{|l|}{ Corticosteroids } \\
\hline$\leq 7.5 \mathrm{mg} / \mathrm{day} *$ & 26.6 & 30.2 & 28.3 \\
\hline$>7.5 \mathrm{mg} / \mathrm{day}^{*}$ & 69.4 & 45.9 & 58.0 \\
\hline Other immunosuppressive agents $\dagger$ & 42.2 & 55.6 & 48.7 \\
\hline Azathioprine & 25.7 & 20.4 & 23.1 \\
\hline Methotrexate & 9.1 & 18.6 & 13.7 \\
\hline Mycophenolate & 6.0 & 16.7 & 11.2 \\
\hline Cyclosporin & 1.5 & 1.7 & 1.6 \\
\hline Leflunomide & 0.6 & 1.3 & 1.0 \\
\hline Antimalarials & 67.2 & 63.4 & 65.3 \\
\hline NSAID & 20.0 & 40.8 & 30.1 \\
\hline HMG CoA reductase inhibitors & 8.2 & 10.1 & 7.6 \\
\hline
\end{tabular}

52 according to the number of score shifts (one to three); significant improvements of two to three letter scores (ie, A to C or D, or B to $\mathrm{D})$ were noted in the $10 \mathrm{mg} / \mathrm{kg}$ group for the musculoskeletal, mucocutaneous and vasculitis domains, and in the $1 \mathrm{mg} / \mathrm{kg}$ group for the musculoskeletal domain. Significantly fewer patients in the belimumab $1 \mathrm{mg} / \mathrm{kg}$ group without a BILAG A score at baseline had worsening in the haematology domain (table 2).

As assessed by SELENA-SLEDAI, significantly more belimumab-treated patients had improvements in the musculoskeletal (1 mg/kg), mucocutaneous (10 mg/kg), and immunological ( 1 and $10 \mathrm{mg} / \mathrm{kg}$ ) domains at week 52 (figure 3A). Post-hoc analysis of the adjusted mean SELENA-SLEDAI score over 52 weeks showed similar results. The mean changes $\pm S E$ in overall adjusted mean SELENA-SLEDAI scores from baseline were significantly better with belimumab $1 \mathrm{mg} / \mathrm{kg}(-2.93 \pm 0.14 ; \mathrm{p}=0.02)$ and $10 \mathrm{mg} /$ $\mathrm{kg}(-3.27 \pm 0.14 ; \mathrm{p}<0.001)$ versus placebo $(-2.57 \pm 0.12)$ over 52 weeks, as well as from weeks 24 to $52(-3.93 \pm 0.17(\mathrm{p}=0.01)$ and $-4.30 \pm 0.18(\mathrm{p}<0.001)$ versus $-3.43 \pm 0.16$, respectively). For individual domains, the changes in adjusted mean SELENA-SLEDAI scores were significantly greater with belimumab 1 and $10 \mathrm{mg} /$ $\mathrm{kg}$ for the musculoskeletal and immunological domains over 52 weeks, and for the immunological domain from weeks 24 to 52. Overall, more patients on belimumab than on placebo had improvement in three or more, as well as two or more, organ systems (figure 3B). Significantly fewer patients in both belimumab groups with no organ domain involvement at baseline had worsening in the immunological $(10 \mathrm{mg} / \mathrm{kg})$, haematologi$\mathrm{cal}(10 \mathrm{mg} / \mathrm{kg})$ and renal $(1 \mathrm{mg} / \mathrm{kg})$ domains (table 2$)$.
$1 \mathrm{~A}$

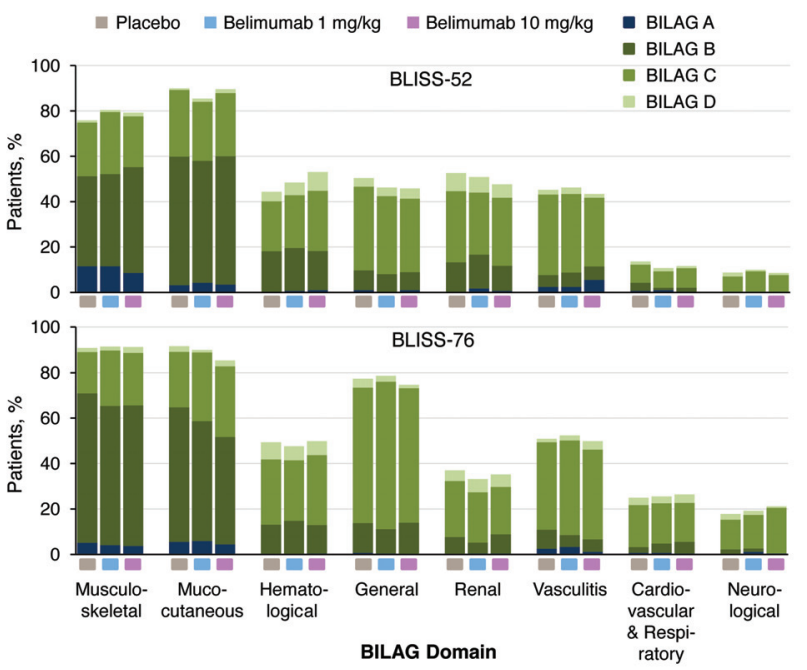

$1 \mathrm{~B}$

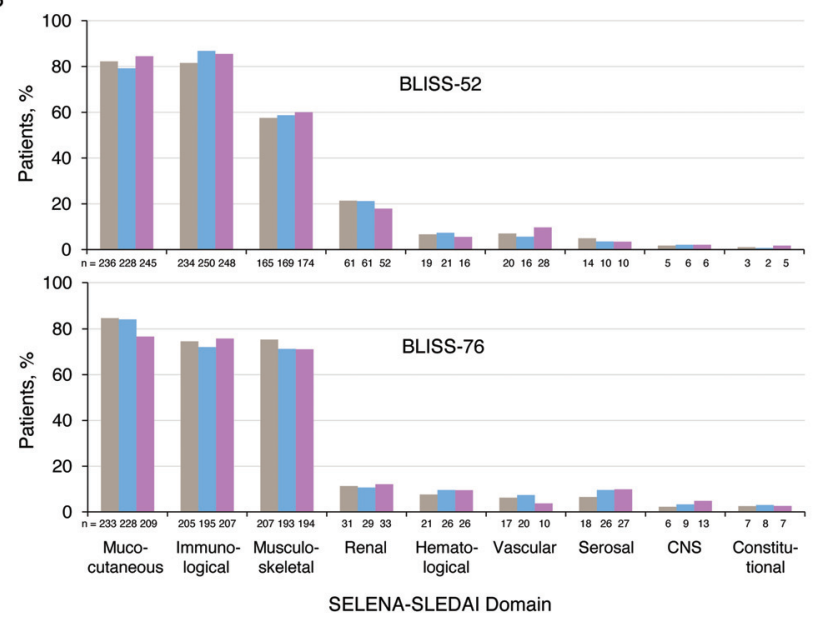

Figure 1 Baseline (A) British Isles Lupus Assessment Group (BILAG) and (B) Safety of Oestrogens in Lupus National Assessment-Systemic Lupus Erythematosus Disease Activity Index (SELENA-SLEDAI) organ involvement in the BLISS-52 and BLISS-76 studies. BILAG involvement is depicted as $A, B, C$ or D score at baseline. CNS, central nervous system.

The most commonly affected individual items in the SELENA-SLEDAI organ domains are presented in figure 3C. In the mucocutaneous domain, improvement rates were observed with belimumab compared with placebo for alopecia, mucosal ulcers and rash. In the musculoskeletal domain, improvement in arthritis was observed with both belimumab doses compared with placebo. Only 12 patients had myositis at baseline, the other item in the musculoskeletal domain. For immunological items, improvement rates (normalisation of serum levels) were greater with both belimumab doses than with placebo for antidsDNA seropositive status and low levels of complement $3(<90$ $\mathrm{mg} / \mathrm{dl})$ or complement $4(<16 \mathrm{mg} / \mathrm{dl})$ at baseline. This treatment effect remained when the SRI was modified not to include SELENA-SLEDAI score contributions from any one of the three most common organ domains (mucocutaneous, musculoskeletal and immunological), and from both the mucocutaneous and musculoskeletal organ domains (data not shown).

Post-hoc analysis of SRI and SELENA-SLEDAI organ system changes in a subgroup of 876 patients with high serological activity (anti-dsDNA positivity and low complement 3/4 levels) 
$2 \mathrm{~A}$

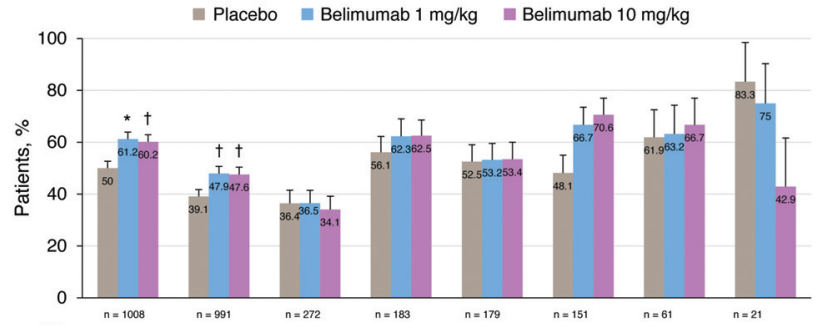

$2 \mathrm{~B}$

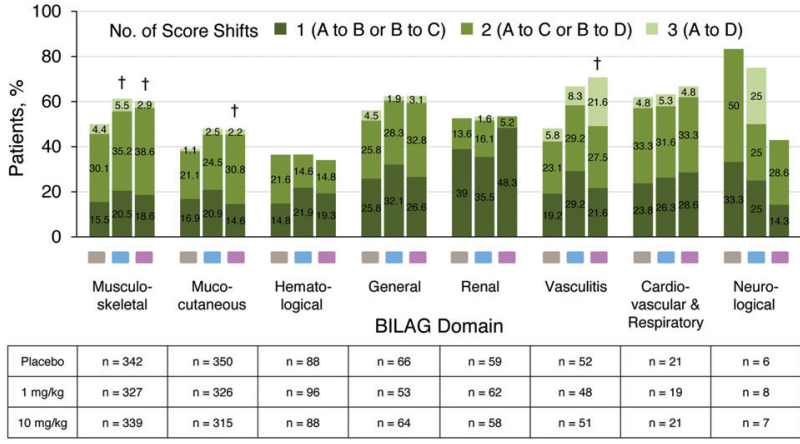

Figure 2 (A) Proportions of patients with improvements from baseline in British Isles Lupus Assessment Group (BILAG) organ domain scores at week 52 (pooled BLISS-52 and BLISS-76 data) among those with an $A$ or $B$ score at baseline. Improvement was defined as a step down from an $A$ or $B$ score to a B, C or D score. (B) Improvement from baseline by one, two, or three-score shift at week 52 in patients with an $A$ or $B$ score at baseline. A one-score shift is a shift from an $A$ score at baseline to a $B$ score at week 52 , or a $B$ score at baseline to a $C$ score at week 52; a two-score shift is from $A$ to $C$ or $B$ to $D$; a three-score shift is from A to $D$. ${ }^{*} p<0.01 ; \dagger p<0.05$.

at baseline demonstrated a greater magnitude of belimumab treatment effect than that observed in all patients. The SRI rates at week 52 were significantly higher with belimumab $1 \mathrm{mg} / \mathrm{kg}$ $(41.5 \% ; \mathrm{p}=0.002)$ and $10 \mathrm{mg} / \mathrm{kg}(51.5 \%$; $\mathrm{p}<0.001)$ than with placebo $(31.7 \%)$. This treatment effect remained when the SRI was modified not to include normalisation of anti-dsDNA and low complement $3 / 4$ in the SELENA-SLEDAI score reduction (data not shown). Highly serologically active patients had greater responses in several organ systems (including mucocutaneous, immunological, musculoskeletal, renal, vasculitis, CNS and constitutional) with belimumab treatment than with placebo (figure 3D). The numbers of organ domains improved by treatment group in the high serological activity subgroup are shown in figure 3E. Overall, significantly more highly serologically active patients on belimumab versus placebo had improvements in a greater number of organ domains (one to three or more), with more patients improving in three or more organ systems on belimumab $1 \mathrm{mg} / \mathrm{kg}$ than on placebo.

In all, 267 patients had SELENA-SLEDAI renal involvement, including 226 with proteinuria (>0.5 g/24 h) and/or haematuria (more than five red blood cells/high-power field; $n=67$ ). In patients with proteinuria greater than $1 \mathrm{~g} / 24 \mathrm{~h}$, the percentage improvements from baseline were significantly greater with belimumab $1 \mathrm{mg} / \mathrm{kg}$ at weeks 24, 28, 32, 40, 44 and 48 than with placebo (all $\mathrm{p}<0.05)$, but there was no significant difference at any time point with belimumab $10 \mathrm{mg} / \mathrm{kg}$.

Among patients with baseline haematological involvement $(\mathrm{n}=137)$, leucopenia was the most common abnormality, with similar rates of baseline grade 1-3 abnormalities across treatment groups $(n=103)$ : improvement rates with placebo and belimumab 1 and $10 \mathrm{mg} / \mathrm{kg}$ were $48.5 \%, 51.4 \%$ and $30.3 \%$, respectively. There were comparable median changes from
Table 2 Rates of worsening in organ domains at 52 weeks

\begin{tabular}{|c|c|c|c|}
\hline \multirow[b]{2}{*}{ Organ domain } & \multicolumn{3}{|c|}{ Standard therapy plus } \\
\hline & Placebo & $\begin{array}{l}\text { Belimumab } \\
1 \mathrm{mg} / \mathrm{kg}\end{array}$ & $\begin{array}{l}\text { Belimumab } \\
10 \mathrm{mg} / \mathrm{kg}\end{array}$ \\
\hline \multicolumn{4}{|c|}{ BILAG: number with worsening/without A domain score at baseline $(\%)^{*}$} \\
\hline Musculoskeletal & $26 / 515(5.0)$ & $20 / 515(3.9)$ & $20 / 528(3.8)$ \\
\hline Mucocutaneous & $24 / 538(4.5)$ & $23 / 531(4.3)$ & $29 / 541(5.4)$ \\
\hline Haematological & $51 / 561(9.1)$ & $31 / 557(5.6) \ddagger$ & $37 / 559(6.6)$ \\
\hline General & $18 / 557(3.2)$ & $16 / 558(2.9)$ & $19 / 560(3.4)$ \\
\hline Renal & $42 / 561(7.5)$ & $27 / 553(4.9)$ & $34 / 560(6.1)$ \\
\hline Vasculitis & $9 / 548(1.6)$ & $2 / 543(0.4)$ & $3 / 544(0.6)$ \\
\hline $\begin{array}{l}\text { Cardiovascular/ } \\
\text { respiratory }\end{array}$ & $5 / 558(0.9)$ & $5 / 554(0.9)$ & $6 / 561(1.1)$ \\
\hline Neurological & $4 / 562(0.7)$ & $3 / 556(0.5)$ & $6 / 562(1.1)$ \\
\hline \multicolumn{4}{|c|}{$\begin{array}{l}\text { SELENA-SLEDAl: number with worsening/with no organ domain involvement at } \\
\text { baseline (\%)* }\end{array}$} \\
\hline Musculoskeletal & $13 / 190(6.8)$ & $12 / 197(6.1)$ & $8 / 195(4.1)$ \\
\hline Mucocutaneous $†$ & $12 / 93(12.9)$ & 14/103 (13.6) & $14 / 109(12.8)$ \\
\hline Immunological & $23 / 123(18.7)$ & $17 / 114(14.9)$ & $8 / 108(7.4) \ddagger$ \\
\hline Haematological & $34 / 522(6.5)$ & 24/512 (4.7) & $17 / 521(3.3) \ddagger$ \\
\hline Constitutional & 4/552 (0.7) & 4/549 (0.7) & 6/551 (1.1) \\
\hline Renal & $40 / 470(8.5)$ & $22 / 469(4.7) \ddagger$ & $31 / 478(6.5)$ \\
\hline Vasculitis $†$ & $2 / 525(0.4)$ & $2 / 523(0.4)$ & $3 / 525(0.6)$ \\
\hline Serositis & $10 / 530(1.9)$ & $4 / 523(0.8)$ & $4 / 526(0.8)$ \\
\hline CNS & $2 / 551(0.4)$ & $3 / 544(0.6)$ & $2 / 544(0.4)$ \\
\hline
\end{tabular}

*For BILAG organ domains, worsening was defined as a step up from an E, D, C or B score to a B or A score. For SELENA-SLEDAl organ domains, worsening was defined as a positive score shift. Missing data were analysed using last observation carried forward.

tIn the original SLEDAl paper, 'mucocutaneous' was denoted as 'dermal'; for purposes of this analysis, 'mucocutaneous' has been used to match BILAG terminology as both terms represent similar cutaneous manifestations. ${ }^{10}$ In addition, the term 'vascular' has been changed to 'vasculitis', because no cerebrovascular accidents were reported and all data refer to cases of vasculitis.

$\ddagger \mathrm{p}<0.05$ versus placebo.

BILAG, British Isles Lupus Assessment Group; CNS, central nervous system;

SELENA-SLEDAI, Safety of Oestrogens in Lupus National Assessment-Systemic Lupus Erythematosus Disease Activity Index.

baseline and proportions of patients with grade $3 / 4$ changes over 52 weeks across the three treatment groups for white blood cells, neutrophils and lymphocytes in both studies. ${ }^{5}$ The 111 patients with baseline vasculitis had improvement rates with placebo and belimumab 1 and $10 \mathrm{mg} / \mathrm{kg}$ of $40.8 \%, 52.8 \%$ and $73.7 \%$, respectively. There was overall improvement with belimumab treatment in the small number of patients with baseline CNS involvement ( $\mathrm{n}=45$ ), with the most common abnormality involved being lupus headache $(n=24)$. Improvement rates for headache with placebo and belimumab 1 and $10 \mathrm{mg} / \mathrm{kg}$ were $20.0 \%, 100 \%$ and $69.2 \%$, respectively.

\section{DISCUSSION}

The present study examined the effects of treatment with belimumab versus placebo, plus standard SLE therapy, on SLE disease activity in specific organ systems in the two phase III trials. Although both SELENA-SLEDAI and BILAG were incorporated in the SRI, the former was used to evaluate efficacy, whereas the latter was used to record organ-specific worsening. In this post-hoc analysis of pooled data from the two phase III trials, the two disease activity instruments were used to evaluate both organ domain improvement and worsening.

The BLISS-52 and BLISS-76 trials, as well as the current posthoc analysis of pooled data, were neither designed nor powered to demonstrate the efficacy of belimumab treatment in individual organ domains. ${ }^{5} 6$ This analysis, however, showed that more patients treated with belimumab than with placebo had 
$3 \mathrm{~A}$

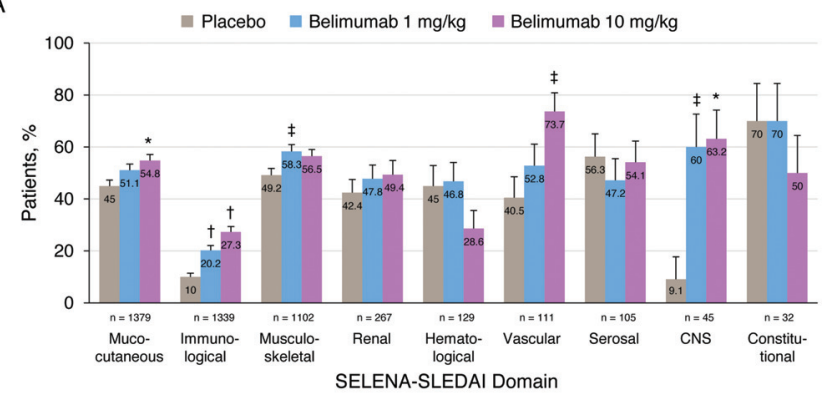

$3 \mathrm{C}$

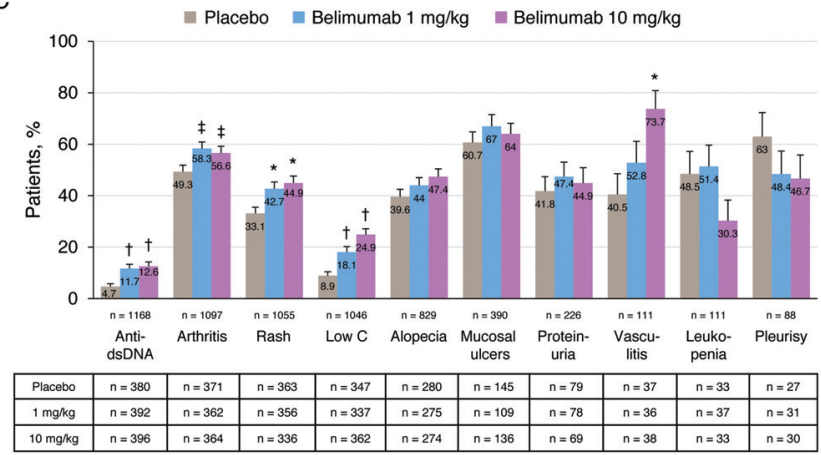

3B

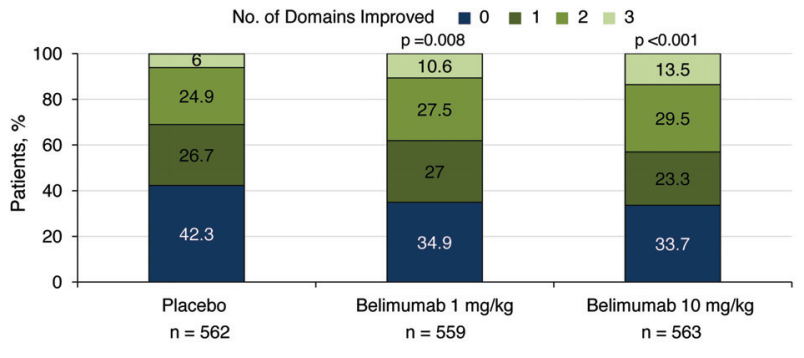

3D

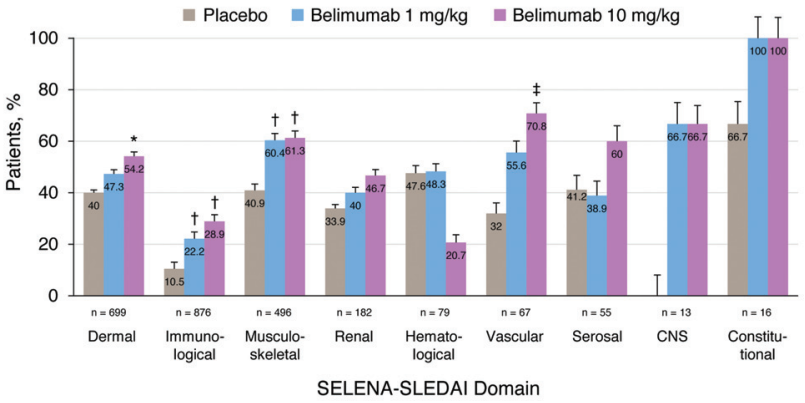

$3 E$

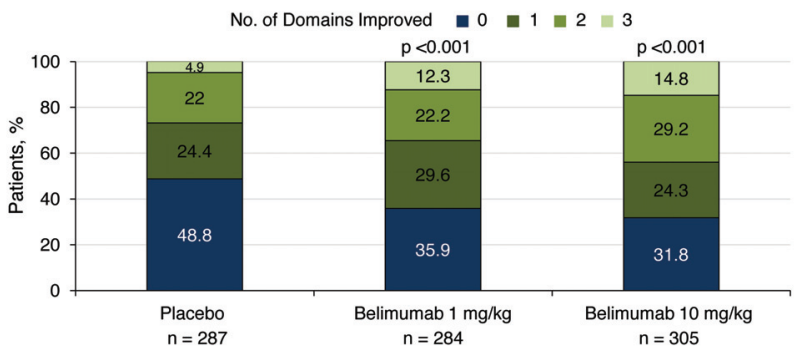

Figure 3 Proportions of patients with improvements from baseline in Safety of Oestrogens in Lupus National Assessment-Systemic Lupus Erythematosus Disease Activity Index (SELENA-SLEDAI) organ domain scores at week 52 in (A) total pooled population and (B) total pooled population according to the number of organ domains improved; (C) proportions in total pooled population with improvements in most common SELENA-SLEDAI individual organ domain item scores; and proportions with improvements in SELENA-SLEDAI organ domain scores at week 52 in (D) subgroup with high serological activity at baseline and (E) subgroup with high serological activity according to the number of organ domains improved. Improvement was defined as negative score shift. ${ }^{*} p<0.01 ; \uparrow p<0.001 ; \ddagger p<0.05$. CNS, central nervous system

improvements, and fewer had worsening across several BILAG and SELENA-SLEDAI organ domains. In general, the findings with BILAG and SELENA-SLEDAI were consistent across the two indices. In particular, both indices indicated more improvement in patients treated with either belimumab dose than with placebo in the mucocutaneous and musculoskeletal domains, which were among the most commonly affected domains at baseline $82 \%$ and $65 \%$ by SELENA-SLEDAI, and $59 \%$ and $60 \%$ by BILAG, respectively). Belimumab treatment was also associated with greater rates of improvement in the SELENA-SLEDAI immunological domain, the second most frequently affected domain on that index. In addition, more patients on belimumab had improvements in three or more organ systems than on standard SLE therapy alone. There were fewer patients treated with belimumab plus standard therapy than standard therapy alone who had worsening in the haematological domains as measured by both SELENA-SLEDAI $(10 \mathrm{mg} / \mathrm{kg}$ ) and BILAG (1 mg/kg), as well as in the immunological domain as measured by SELENA-SLEDAI (10 mg/kg).

Improvement was noted in vasculitis with both disease activity indices. Conclusions regarding improvement or worsening in rare organ manifestations are, however, limited by the small sample sizes. Vasculitis at baseline was described as primarily cutaneous based on BILAG items scored at baseline. Furthermore, for the CNS and renal domains, conclusions are limited by the exclusion of patients with severe lupus nephritis or severe CNS manifestations from the two trials. ${ }^{56}$

The haematological domain showed similar rates of improvement in all three treatment groups as measured by BILAG, and in the placebo and belimumab $1 \mathrm{mg} / \mathrm{kg}$ group as measured by SELENA-SLEDAI. The rate of response with belimumab $10 \mathrm{mg} /$ $\mathrm{kg}$ was lower than with placebo, although there were similar proportions of patients with grades 1-4 leucopenia, lymphopenia, neutropenia and thrombocytopenia at baseline, and after 52 weeks of therapy. The lower response in the $10 \mathrm{mg} / \mathrm{kg}$ group was not significantly different and was most likely due to the small sample size with haematological involvement ( $\mathrm{n}=129$; $7.7 \%$ of the total population), and may be related to the higher proportion of patients with belimumab versus placebo who had leucopenia at baseline and were on immunosuppressive agents (63\% vs $36 \%$ ).

A limitation of this post-hoc analysis is the small sample size of some organ system manifestations and not correcting 
for multiple comparisons. These two factors could have led to spurious results with regard to improvement or worsening, particularly in the organ systems with less than $10 \%$ of the total population. Further study in these less common manifestations would be needed to evaluate the potential belimumab treatment effect properly.

Multivariate analysis of the pooled BLISS-52 and BLISS-76 data identified high disease activity (SELENA-SLEDAI score $\geq 10$ ), anti-dsDNA positivity, low complement 3 or 4 levels and prednisone use at baseline as being predictors of a better SRI response with belimumab plus standard therapy than with standard therapy alone. ${ }^{7}$ Post-hoc analysis suggested that patients with high serological activity (ie, both anti-dsDNA positivity and low complement 3 or 4 levels; $52 \%$ of the study population) have higher disease activity, and may have greater magnitudes of SRI response and individual organ domain improvement with belimumab than with standard therapy alone. These results are consistent with the improvement noted in the immunological domain (decrease in anti-dsDNA autoantibodies, or increase in complement 3 or 4 levels), as well as the reductions in other autoantibodies, or selected B-cell and plasma cell subsets observed in the BLISS trials. ${ }^{13}$ Although the most commonly affected organ domains (ie, mucocutaneous, musculoskeletal and immunological) improved with belimumab therapy, there were also responses detected in the CNS, renal and vasculitis domains that are often associated with high disease activity (SELENA-SLEDAI score $\geq 10$ ), which supports the observations in the multivariate analysis.?

In conclusion, a post-hoc analysis of patients from the BLISS52 and BLISS-76 trials found that treatment with belimumab plus standard SLE therapy was effective in the organ systems most commonly affected at study entry, including the musculoskeletal, mucocutaneous and immunological domains. The treatment effect with belimumab remained after removal of the contribution of these three common organ domains, or both the musculoskeletal and mucocutaneous domains, to SELENASLEDAI scoring. These results demonstrate that the responses observed in the two phase III studies were not driven by effects on a single organ domain. Rather belimumab had effects on multiple organ systems.

Contributors All authors have read and approved the manuscript. SM, JTM, RF, SVN, EMG and MAP participated in study design, data acquisition, analysis, and interpretation and manuscript drafting and revision. JSG was involved with data acquisition and interpretation, and manuscript drafting and revision. DG and DPD participated in data acquisition, and manuscript drafting and revision. $A D$ was involved with data interpretation, and manuscript drafting and revision. SC, ZJZ, $\mathrm{DH}$ and WF participated in study design, data analysis and interpretation, and manuscript drafting and revision.
Acknowledgements Editorial support (writing assistance, assembling tables and figures, collating author comments, grammatical editing, and referencing) was provided by Matt Stenger and Eleanore Gross of BioScience Communications, New York, NY, USA.

\section{Collaborators BLISS-52 and BLISS-76 Study Groups.}

Funding This study was supported by Human Genome Sciences, Rockville, Maryland, USA, and GlaxoSmithKline, Uxbridge, UK.

Competing interests SM has received grant support and payment for board membership from Human Genome Sciences. JTM and EMG have received consulting fees and grant support from HGS and GlaxoSmithKline. RF has received research or grant support, travel support, and payment for review activities, board membership, and consultancy from HGS and GSK. SVN has received consulting fees and travel support from HGS. DG has received consulting fees from HGS and GSK. DPD has received payment for board membership and consultancy from GSK. AD has received consulting and speaking fees from GSK. SC, ZJZ, DH and WF are employed by and own stock in HGS MAP has received consulting fees from HGS and GSK, and is a member of their advisory boards. All other authors have no competing interests to disclose.

Provenance and peer review Not commissioned; externally peer reviewed.

\section{REFERENCES}

1. Lau CS, Mak A. The socioeconomic burden of SLE. Nat Rev Rheumatol 2009:5:400-4.

2. Rahman A, Isenberg DA. Systemic lupus erythematosus. N Engl J Med 2008:358:929-39.

3. Baker KP, Edwards BM, Main SH, et al. Generation and characterization of LymphoStat-B, a human monoclonal antibody that antagonizes the bioactivities of B lymphocyte stimulator. Arthritis Rheum 2003:48:3253-65.

4. Petri M, Stohl W, Chatham W, et al. Association of plasma B lymphocyte stimulator levels and disease activity in systemic lupus erythematosus. Arthritis Rheum 2008;58:2453-9.

5. Furie R, Petri M, Zamani 0, et al. A phase III, randomized, placebo-controlled study of belimumab, a monoclonal antibody that inhibits B lymphocyte stimulator, in patients with systemic lupus erythematosus. Arthritis Rheum 2011;63:3918-30.

6. Navarra SV, Guzmán RM, Gallacher AE, et al. Efficacy and safety of belimumab in patients with active systemic lupus erythematosus: a randomised, placebo-controlled, phase 3 trial. Lancet 2011;377:721-31.

7. van Vollenhoven RF, Petri MA, Cervera R, et al. Belimumab in the treatment of systemic lupus erythematosus: high disease activity predictors of response. Ann Rheum Dis 15 February 2012 doi:10.1136/annrheumdis-2011-200937

8. Furie RA, Petri MA, Wallace DJ, et al. Novel evidence-based systemic lupus erythematosus responder index. Arthritis Rheum 2009;61: 1143-51.

9. Petri M, Kim MY, Kalunian KC, et al. Combined oral contraceptives in women with systemic lupus erythematosus. N Engl J Med 2005;353:2550-8.

10. Isenberg DA, Gordon C. From BILAG to BLIPS - disease activity assessment in lupus past, present and future. Lupus 2000;9:651-4.

11. Bombardier C, Gladman DD, Urowitz MB, et al. Derivation of the SLEDAI. A disease activity index for lupus patients. The Committee on Prognosis Studies in SLE. Arthritis Rheum 1992;35:630-40.

12. Touma Z, Urowitz MB, Ibañez D, et al. SLEDAI-2K 10 days versus SLEDAI-2K 30 days in a longitudinal evaluation. Lupus 2011;20:67-70.

13. Stohl W, Jacob N, Guo S, et al. Constitutive overexpression of BAFF in autoimmune-resistant mice drives only some aspects of systemic lupus erythematosus-like autoimmunity. Arthritis Rheum 2010;62:2432-42. 